\section{FRI0478 SUBCHONDRAL BONE SCLEROSIS ON COMPUTED TOMOGRAPHY - DOES IT HAVE ANY VALUE IN THE DIAGNOSIS OF INFLAMMATORY SACROILIITIS OR IS IT A NON-SPECIFIC FINDING?}

O. Azmat ${ }^{1}$, R.G. Lambert ${ }^{1}$, Z. Jibri ${ }^{1}$, W.P. Maksymowych ${ }^{2} .{ }^{1}$ Radiology; ${ }^{2}$ Medicine, University of Alberta, Edmonton, Canada

Background: Sclerosis in the sacroiliac joints (SIJ) on radiography and computed tomography (CT) is common but widely considered a non-specific finding of sacroilitis due to an association with degeneration and osteitis condensans ilii, despite little formal study. Availability of low dose radiation CT may lead to more widespread use for diagnostic evaluation.

Objectives: We standardized the definition of sclerosis on CT and then aimed to determine whether this lesion could be reliably detected and its diagnostic utility. Methods: 215 CT scans were obtained from patients with a history of low back pain. 107 patients had a clinical diagnosis of spondyloarthritis (SpA) and 108 patients were clinically proven not to have SpA. Groups were age and gender matched ( 140 males, 75 females, mean age was 45 years). Three musculoskeletal radiologists, blinded to patient demographics and diagnosis, scored the CTs after standardization of lesion definitions and calibration. Erosions, sclerosis, and ankylosis were graded by size and number of articular surfaces/ joints involved. Sclerosis was considered definite if located along the cartilaginous compartment, measured $>5 \mathrm{~mm}$ in all 3 planes, and present $>5 \mathrm{~mm}$ from the joint surface. Discrepant scores were arbitrated and inter-reader reliability calculated by intra-class correlation coefficient (ICC). Diagnostic utility of CT lesions was determined by calculating sensitivity and specificity for the clinical diagnosis and by logistic regression.

Results: ICC for sclerosis and erosion for each articular surface ranged from $0.65-0.76$ and $0.71-0.78$, respectively. ICC for ankylosis was $0.87-0.89$. Sclerosis occurred in $87(81 \%)$ cases with SpA and $25(23 \%)$ controls. For a single articular surface the specificity for sacroilitis ranged between $88-94 \%$, for any two articular surfaces $95-100 \%$, for all 4 articular surfaces $100 \%$. Sensitivity ranged from $14 \%$ (4 articular surfaces) to $55 \%$ (either ilium). Erosion and ankylosis had a similar specificity range of $91-100 \%$ and $92-93 \%$. The odds ratio was 4.9 for presence of definite sclerosis, and 12.6 for bilateral joint involvement. The odds ratio increased to 84.2 for bilateral erosion and 22.8 for bilateral ankylosis.

Conclusions: When sclerosis measures $>5 \mathrm{~mm}$ in three planes and is located $>5 \mathrm{~mm}$ from a joint perimeter, it has high specificity for sacroilitis, regardless of how many articular surfaces are involved, with similar specificity to erosion and ankylosis.

Disclosure of Interest: None declared

DOI: 10.1136/annrheumdis-2017-eular.6292

\section{FRI0479 PREDICTORS OF LONG-TERM MODIFIED MINIMAL DISEASE ACTIVITY RESPONSE IN PERIPHERAL SPONDYLOARTHRITIS PATIENTS TREATED WITH ADALIMUMAB}

L.C. Coates ${ }^{1}$, S. Abraham ${ }^{2}$, W. Tillett $^{3}$, P.J. Mease ${ }^{4}$, S. Ramiro ${ }^{5}$, T. Wu ${ }^{6}$, X. Wang ${ }^{6}$, A.L. Pangan ${ }^{6}$, I.-H. Song ${ }^{6} .{ }^{1}$ University of Leeds and Leeds Teaching Hospitals NHS Trust, Leeds; ${ }^{2}$ NIHR/Wellcome CRF, Imperial College Healthcare NHS Trust, London; ${ }^{3}$ Royal National Hospital for Rheumatic Diseases and University of Bath, Bath, United Kingdom: ${ }^{4}$ Swedish Medical Center and University of Washington, Seattle, United States; ${ }^{5}$ Leiden University Medical Center, Leiden, Netherlands; ${ }^{6}$ AbbVie Inc., N Chicago, United States

Background: There is a lack of validated outcome measures in non-psoriatic peripheral spondyloarthritis ( $\mathrm{pSpA})$. Therefore, a modified version of the minimal disease activity $(\mathrm{mMDA})^{1}$ was developed and validated. Identification of factors that predict long-term mMDA response in pSpA patients (pts) can facilitate decisions regarding treatment initiation and maintenance.

Objectives: The purpose of this analysis was to determine predictors of long-term mMDA response following adalimumab (ADA) treatment in pSpA pts from the ABILITY-2 study.

Methods: ABILITY- $2^{2}$ was a phase 3 randomized, double-blind trial evaluating the efficacy and safety of $40 \mathrm{mg}$ ADA every other week versus placebo (PBO) over 12 weeks (wks) followed by open-label (OL) ADA for 144 wks in pts with pSpA. This post-hoc analysis included pts who received at least one dose of ADA during the PBO-controlled period or OL extension. The mMDA for PSpA was defined as achieving at least 5 out of the following 6 criteria: 1) TJC78 $\leq 1$; 2) SJC76 $\leq 1$; 3) pt pain visual analog scale (VAS) $\leq 15$ of $100 \mathrm{~mm}$; 4) pt global activity

Abstract FRI0478 - Table

Sclerosis of articular surface (s) involved

Single articular surface

Any 2 articular surfaces All 4 articular surfaces

Erosion of articular surface (s) involved

Single articular surface

Any 2 articular surfaces All 4 articular surfaces

Ankylosis of articular surface (s) involved

Single joint

Both joints
Either ilium: 87\% (82-91) - 91\% (87-95)

Either sacrum: 93\% (89-96) - 94\% (90-97)

$96 \%(94-99)-99 \%(98-100)$ $99 \%(8-100)$

Specificity $(95 \% \mathrm{Cl})$

Either sacrum: 94\% (91-98)

$96 \%(93-99)-100 \%(100-100)$

$100 \%(100-100)$

Specificity $(95 \% \mathrm{Cl})$

$92 \%(88-95)$

$93 \%(89-96)$
Either ilium: 90\% (85-94)
(PtGA) VAS $<20$ of $100 \mathrm{~mm}$; 5) HAQ-DI $<0.5$; and 6) tender entheseal points $\leq 1$ (Leeds Enthesitis Index [LEI] or Spondyloarthritis Research Consortium of Canada [SPARCC] Enthesitis Index). In this post hoc analysis, multiple logistic regression with stepwise variable selection was used to determine predictors of long-term (yrs 1-3) and sustained (defined as mMDA for at least 24 consecutive wks) mMDA responses. Variable selection of baseline (BL) pt demographics and disease characteristics were performed with and without mMDA response at wk 16 (mMDA16) as a candidate. In pts achieving mMDA at wk 16, ADA exposure ranged between 4 and 16 wks.

Results: In pSpA pts treated with ADA, mMDA (5/6 LEI or SPARCC) was achieved by almost $41 \%, 49 \%$, and $50 \%$ of pts at yrs 1,2 , and 3 , respectively and sustained mMDA response was achieved by $42 \%$ of pts. Regardless of mMDA definition, achieving mMDA response at wk 16 (up to 16 wks of ADA) was a robust positive predictor of attaining both long-term mMDA at yrs 1-3 and sustained mMDA (Figure). In the model examining the BL predictors (model without mMDA16), age, BL enthesitis and BL BASDAl scores were most commonly selected as negative predictors for achieving long-term and sustained mMDA. Other selected predictors included BL dactylitis, physician's global assessment, hsCRP, and male sex; however, these predictors were not consistently selected for all time points or sustained mMDA.

Conclusions: Early mMDA response is a stronger and more consistent predictor of long-term mMDA, whether at 1, 2, or 3 yrs or sustained over time, than BL

Figure Odds Ratio for Predictors of Longterm (years 13) and Sustaine mMDA responses*
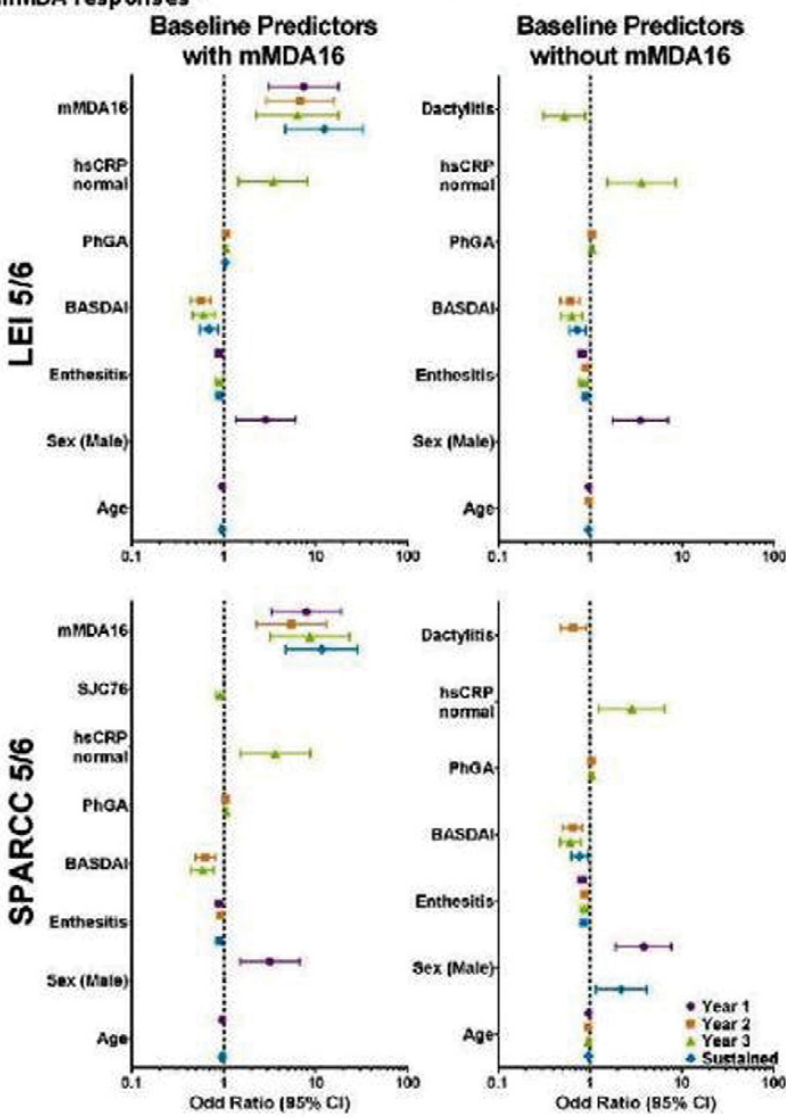

"Only variables selected by stepwise selection model are shown (model selected variables are significant at $P<05$ )

MMDA16 = modified minimal disease actwity response at week $16 ; \mathrm{LEI}=$ Leeds enthesitis index; SPARCC $=$ Spondyloarthritis Research Consortium of Canada enthesitis index, hsCRP = high sensitvity C-reactwe protein; PhOA = physician's global assessment of disease activity, BASDAI = Bath ankylosing spondylitis disease actwity index; $S \mathrm{JC} 76=$ snollen joint court 76 ioints): $\mathrm{Cl}=$ confidence intervat
Sensitivity $(95 \% \mathrm{Cl})$

Either ilium: 46\% (39-52) - 51\% (44-58)

Either sacrum: $19 \%(14-25)-21 \%(16-26)$

$10 \%(6-14)-41 \%(35-48)$

$14 \%(9-19)$

Sensitivity $(95 \% \mathrm{Cl})$

Either ilium: $77 \%$ (71-83)

Either sacrum: $65 \%(58-72)$

$24 \%(18-31)-67 \%(60-74)$

$38.1 \%(31-45)$

Sensitivity $(95 \% \mathrm{Cl})$

$60 \%(53-66)$

$58 \%(51-64)$ 